\title{
Rare weak decays and nuclear structure
}

\author{
Jouni Suhonen ${ }^{1, a}$ \\ ${ }^{1}$ Department of Physics, University of Jyväskylä, P.O. Box 35 (YFL), Fl-40014, Jyväskylä, Finland
}

\begin{abstract}
Weak interactions cause the atomic nuclei to decay via beta and double beta decays. Double beta decays are extremely rare since they are weak-interaction processes of the second order. Also (single) beta decays can be extremely rare. This can be caused by either a large difference between the spins of the initial and final state (the so-called "forbidden" beta decays) or an extremely small $Q$ value (decay energy) of the decay. All these cases are discussed in this article, and particular emphasis is given to the neutrinoless double electron capture on the double beta side of decays.
\end{abstract}

\section{Introduction}

Weak interactions, as the name indicates, are indeed weak if we measure weakness in terms of time scales of processes they generate, say in atomic nuclei where they prompt disintegration phenomena in the time scale of seconds. However, notable exceptions to this state of affairs are caused by (a) extremely small decay energies (Q-values), (b) initial and final nuclear states with large difference in angular momentum and (c) weak-interaction processes of higher order (for reviews see e.g. [1-3]). These extreme conditions of decay lead to processes that involve time scales far beyond the seconds scale, to scales much longer than the age of the Universe. Typically such processes have half-lives of the order of $10^{20}$ years (practically the age of the Universe squared!) and thus can be called 'ultra slow'. The related transitions need special experimental facilities and dedicated experimental techniques in order to be detected. The detection sites of such rare processes need to be protected against cosmic rays, i.e. the flux of particles from outer space. This is why the dedicated experiments go underground, in deep mine shafts or under huge amounts of massive mountain rock. Hence the related scientific effort is appropriately called "underground physics", or to contrast it with the research done in particle accelerator facilities, "non-accelerator physics".

Examples of the above-mentioned classes of rare decays are the "ultra-low" $Q$-value decays examined lately both in Penning-trap measurements and some some underground facilities (see Sect. 2), decays that involve large differences in angular momentum, like the $\beta^{-}$decay of ${ }^{96} \mathrm{Zr}$ that competes with the double beta decay of the same nucleus (see Sect. 3) and neutrinoless double electron-capture decays presented in Sect. 4.

\section{Decays with ultra-low $Q$ values}

During the last decade a lot of information on the relative masses and mixing of neutrinos has been gained. A still missing piece of information is the absolute mass scale of the neutrinos which is

\footnotetext{
ae-mail: jouni.suhonen@phys.jyu.fi
} 
currently being investigated in experiments that focus on the nuclear double beta decay [1] of various nuclear systems [4] or to the $\beta^{-}$decays of tritium (the KATRIN experiment [5]) and ${ }^{187} \operatorname{Re}$ (the MARE experiment [6]). The latter two experiments are direct searches of the electron-neutrino mass via the slight distortion of the electron end-point spectrum. To detect this distortion, as small as possible $Q$ value of the decay is desirable.

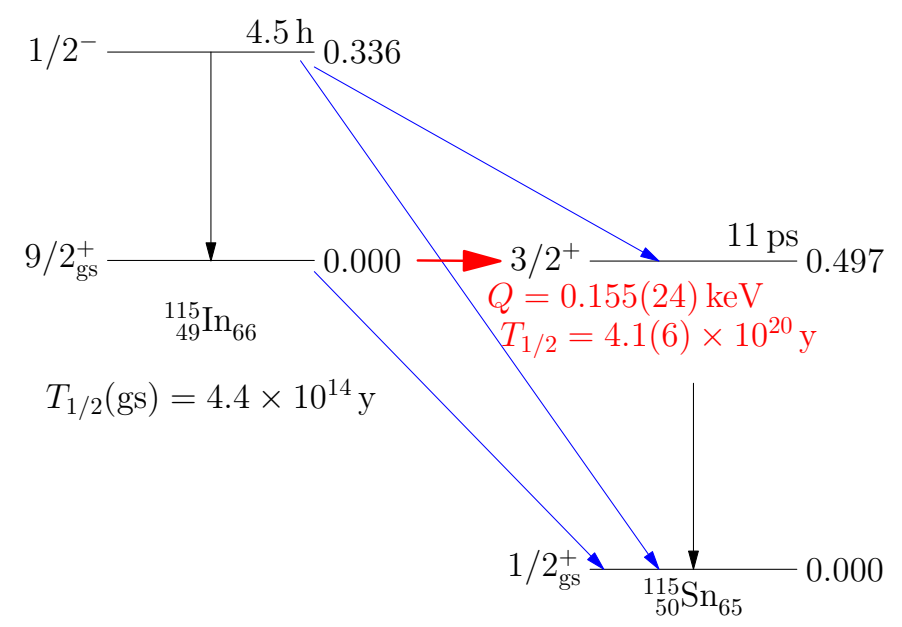

Figure 1. $\beta^{-}$-decay transitions from the ground state of ${ }^{115} \mathrm{In}$ to the ground state and first excited state in ${ }^{115} \mathrm{Sn}$. The numbers to the right of the energy levels are excitation energies in $\mathrm{MeV}$.

There are some other very interesting cases of potential neutrino-mass studies. One of them is the $\beta^{-}$decay of the $9 / 2^{+}$ground state of ${ }^{115} \mathrm{In}$ to the first excited state of ${ }^{115} \mathrm{Sn}$ with spin-parity $3 / 2^{+}$(see figure 1). This decay transition is second-forbidden unique so that the line shape of the decay is simple (for reviews of the formalism of $\beta^{-}, \beta^{+}$and electron-capture (EC) decays see [7-10]). Interesting about this decay transition is that it has a world-record small $Q$ value of $0.155(24) \mathrm{keV}$ [11] so that it can be called "ultra-low" (i.e. well below $1 \mathrm{keV}$ ). Measurement of such a small $Q$ value is based on the Penning trap techniques $[11,12]$. The corresponding decay branch was measured by the HADES underground facility in Belgium to have a partial half-life of 4.1(6) $\times 10^{20} \mathrm{yr}$ [12] and it has been speculated that the decay branch could be used as a neutrino-mass detector [13]. Even more intriguing is that such an ultra-low $Q$ value seems to enhance the interference of atomic effects in the nuclear decay, as discussed in $[14,15]$.

An other potential case for such an ultra-low $Q$-value decay is shown in figure 2 . There the $7 / 2^{+}$ ground state of ${ }^{135} \mathrm{Cs} \beta^{-}$decays to the first and second excited state of ${ }^{135} \mathrm{Ba}$ with spin-parities $1 / 2^{+}$ and $11 / 2^{-}$which are second-forbidden and first-forbidden unique decays, respectively [16]. The halflife corresponding to the second-forbidden non-unique decay transition to the ground state of ${ }^{135} \mathrm{Ba}$ can be computed by allowing the decay energy $Q$ and the axial-vector coupling constant $g_{\mathrm{A}}$ vary in value. This produces the shaded area of computed half-life values in figure 3 . There are also two half-life and $Q$-value measurements $[17,18]$ that are in strong tension with each other, as shown in figure 3. Depending on which one of the measurements is correct, either the decay to the first or to the second excited state can produce a transition with an ultra-low $Q$ value [16]. So, accurate Penning trap measurement of the mass difference between ${ }^{135} \mathrm{Cs}$ and ${ }^{135} \mathrm{Ba}$ is called for. 
INPC 2013

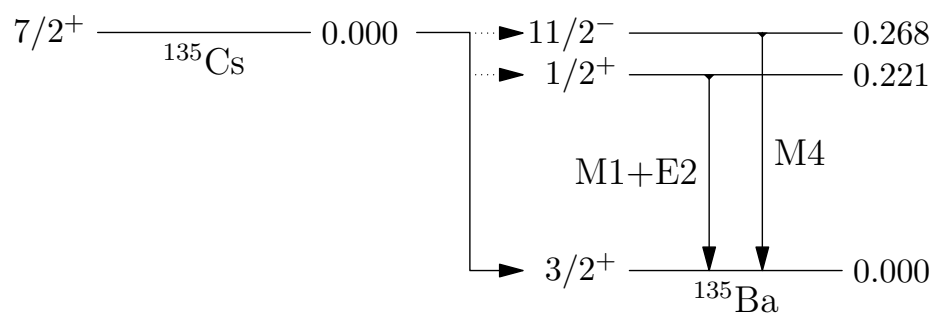

Figure 2. $\beta^{-}$-decay transitions from the ground state of ${ }^{135} \mathrm{Cs}$ to the ground state and first two excited states in ${ }^{135} \mathrm{Ba}$. The numbers to the right of the energy levels are excitation energies in $\mathrm{MeV}$.

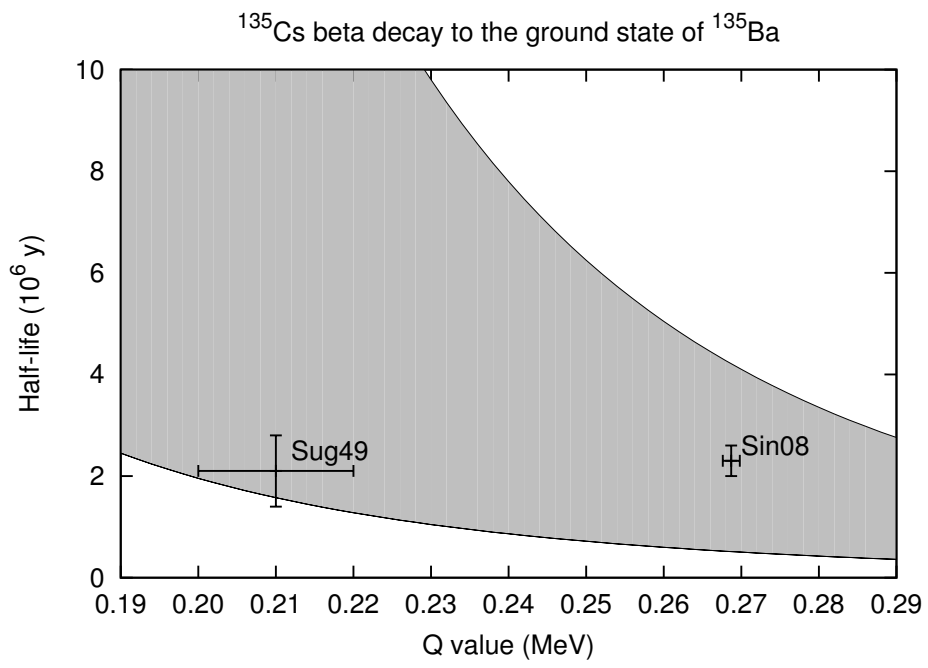

Figure 3. Computed $\beta^{-}$-decay half-life as a function of the decay $Q$ value for the transition from ${ }^{135} \mathrm{Cs}$ to the ground state of ${ }^{135} \mathrm{Ba}$ for the range $g_{\mathrm{A}}=1.00-1.25$ (shaded area). Two experimental half-life and $Q$-value measurements $[17,18]$ are also given.

Yet another potential ultra-low $Q$-value decay is presented in figure 4 . This is the fourth-forbidden non-unique $\beta^{-}$decay of the $1 / 2^{+}$ground state of ${ }^{115} \mathrm{Cd}$ to the second excited $9 / 2^{+}$state in ${ }^{115} \mathrm{In}$. Again, the energy difference of the ground states of the mother and daughter nuclei is not known accurately enough to tell whether this transition is possible or not, but if the $Q$ value is positive, it is most likely ultra-low. A thorough treatment of this transition, and also the other transitions of figure 4 has been performed in [19].

In table 1 are listed some other potential ultra-low $Q$-value transitions in nuclei with mass numbers $A \leq 161$ [20]. All the initial states of the first column of the table are ground states of the respective nuclei. The second column indicates the final nucleus and its final state with its excitation energy given in the third column with the experimental error included in the parenthesis. The fourth column of the table indicates the beta-decay type and the last column lists the experimental decay $Q$ value derived from the experimental mass difference between the mother and daughter nuclei and the measured excitation energy of the final state. In the table the decay type is either $\beta^{-}$or electron capture (EC). 


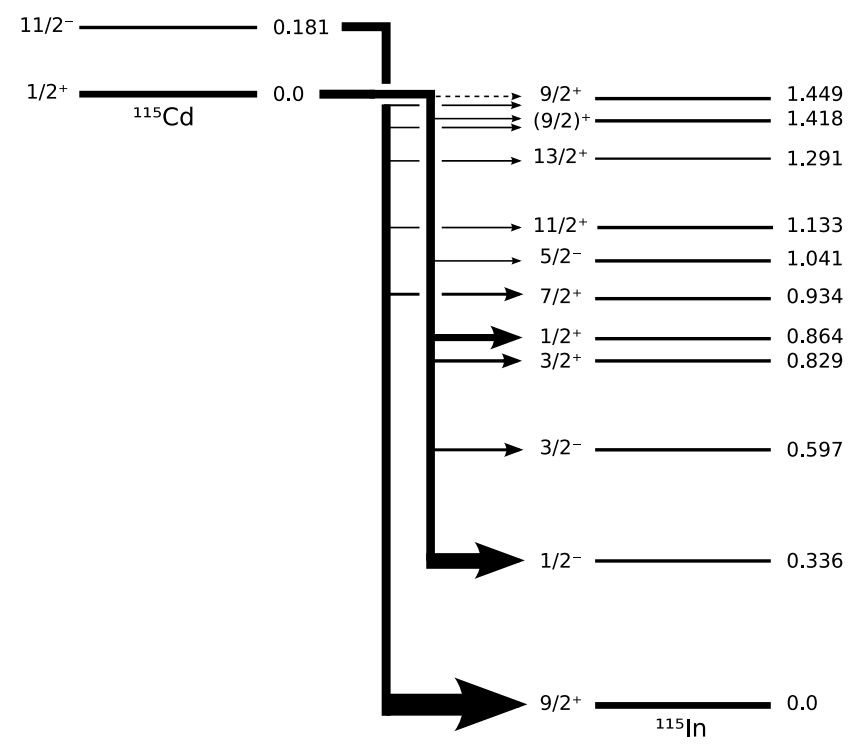

Figure 4. The $\beta^{-}$decay scheme of ${ }^{115} \mathrm{Cd}$. Shown are the decay transitions from the ground state and the isomeric first excited state of ${ }^{115} \mathrm{Cd}$ to states of the daughter nucleus ${ }^{115} \mathrm{In}$ with experimentally measured branching ratios. The dominant branches are indicated by thicker arrows. The dashed line shows the possible ultra-low-Q-value branch $1 / 2^{+} \rightarrow 9 / 2^{+}$. The numbers to the right of the energy levels are excitation energies in $\mathrm{MeV}$.

Table 1. Potential candidate transitions with ultra-low $Q$ values. The first column gives the initial ground state of the listed nucleus and the second column the final excited state of the listed nucleus. The third column gives the experimental excitation energy with the experimental error. The fourth column gives the decay type and the last column the derived experimental decay $Q$ value in units of $\mathrm{keV}$.

\begin{tabular}{ccccc}
\hline initial state & final state & $E^{*}$ in $\mathrm{keV}$ & decay type & $Q[\mathrm{keV}]$ \\
\hline${ }^{77} \mathrm{As}\left(3 / 2^{-}\right)$ & ${ }^{77} \mathrm{Se}\left(5 / 2^{+}\right)$ & $680.1046(16)$ & $1^{\text {st }}$ non-unique $\beta^{-}$ & $2.8 \pm 1.8$ \\
${ }^{111} \mathrm{In}\left(9 / 2^{+}\right)$ & ${ }^{111} \mathrm{Cd}\left(3 / 2^{+}\right)$ & $864.8(3)$ & $2^{\text {nd }}$ unique EC & $-2.8 \pm 5.0$ \\
& ${ }^{111} \mathrm{Cd}\left(3 / 2^{+}\right)$ & $866.60(6)$ & $2^{\text {nd }}$ unique EC & $-4.6 \pm 5.0$ \\
${ }^{131} \mathrm{I}\left(7 / 2^{+}\right)$ & ${ }^{131} \mathrm{Xe}\left(9 / 2^{+}\right)$ & $971.22(13)$ & allowed $\beta^{-}$ & $-0.4 \pm 0.7$ \\
${ }^{146} \mathrm{Pm}\left(3^{-}\right)$ & ${ }^{146} \mathrm{Nd}\left(2^{+}\right)$ & $1470.59(6)$ & $1^{\text {st }}$ non-unique EC & $1.4 \pm 4.0$ \\
${ }^{149} \mathrm{Gd}\left(7 / 2^{-}\right)$ & ${ }^{149} \mathrm{Eu}\left(5 / 2^{+}\right)$ & $1312(4)$ & $1^{\text {st }}$ non-unique EC & $1 \pm 6$ \\
${ }^{155} \mathrm{Eu}\left(5 / 2^{+}\right)$ & ${ }^{155} \mathrm{Gd}\left(9 / 2^{-}\right)$ & $251.7056(10)$ & $1^{\text {st }}$ unique $\beta^{-}$ & $1.0 \pm 1.2$ \\
${ }^{159} \mathrm{Dy}\left(3 / 2^{-}\right)$ & ${ }^{159} \mathrm{~Tb}\left(5 / 2^{-}\right)$ & $363.5449(14)$ & allowed EC & $2.1 \pm 1.2$ \\
${ }^{161} \mathrm{Ho}\left(7 / 2^{-}\right)$ & ${ }^{161} \mathrm{Dy}\left(7 / 2^{-}\right)$ & $857.502(7)$ & allowed EC & $1.4 \pm 2.7$ \\
& ${ }^{161} \mathrm{Dy}\left(3 / 2^{-}\right)$ & $858.7919(18)$ & $2^{\text {nd }}$ non-unique EC & $0.1 \pm 2.7$ \\
\hline
\end{tabular}

In table 1 there is only one initial nucleus that is odd-odd $\left({ }^{146} \mathrm{Pm}\right)$, all the others are odd-mass nuclei. Particularly interesting cases are the ones with allowed Gamow-Teller $\beta^{-}\left({ }^{131} \mathrm{I}\right)$ or allowed Gamow-Teller $\left({ }^{159} \mathrm{Dy}\right)$ and Gamow-Teller/Fermi $\left({ }^{161} \mathrm{Ho}\right)$ electron-capture decays. Also the firstforbidden unique $\beta^{-}$decay $\left({ }^{155} \mathrm{Eu}\right)$ and the second-forbidden unique electron-capture decays $\left({ }^{111} \mathrm{In}\right)$ are of high interest. The rest are non-unique decays and depend on several nuclear matrix elements 
without a unique line shape. The allowed and first-forbidden unique $\beta^{-}$transitions $\left({ }^{131} \mathrm{I}\right.$ and $\left.{ }^{155} \mathrm{Eu}\right)$ are optimal for the experimental identification of the neutrino-mass distortions at the end point of the beta spectrum. These can help in determining the absolute neutrino mass in beta-decay experiments. For the investigation of the electron-cloud-nucleus interference effects the allowed, first-forbidden unique and second-forbidden unique $\beta^{-}$and electron-capture decays are very useful. In particular, for the EC decays with ultra-low $Q$ values the electron-screening effects become of paramount importance.

From the nuclear-structure point of view the first three nuclei of table 1 are (nearly) spherical or weakly deformed whereas the heavier nuclei are well deformed and require a deformed mean field as the starting point in nuclear-structure calculations.

Finally, It should be stated that verification of the spin-parities of some of the final states in table 1 has to be done by nuclear-spectroscopy methods. At the same time it is imperative to perform highprecision Penning-trap measurements to improve the accuracy of the mass differences of the involved nuclei.

\section{Competition of beta and double beta decays in ${ }^{96} \mathrm{Zr}$}

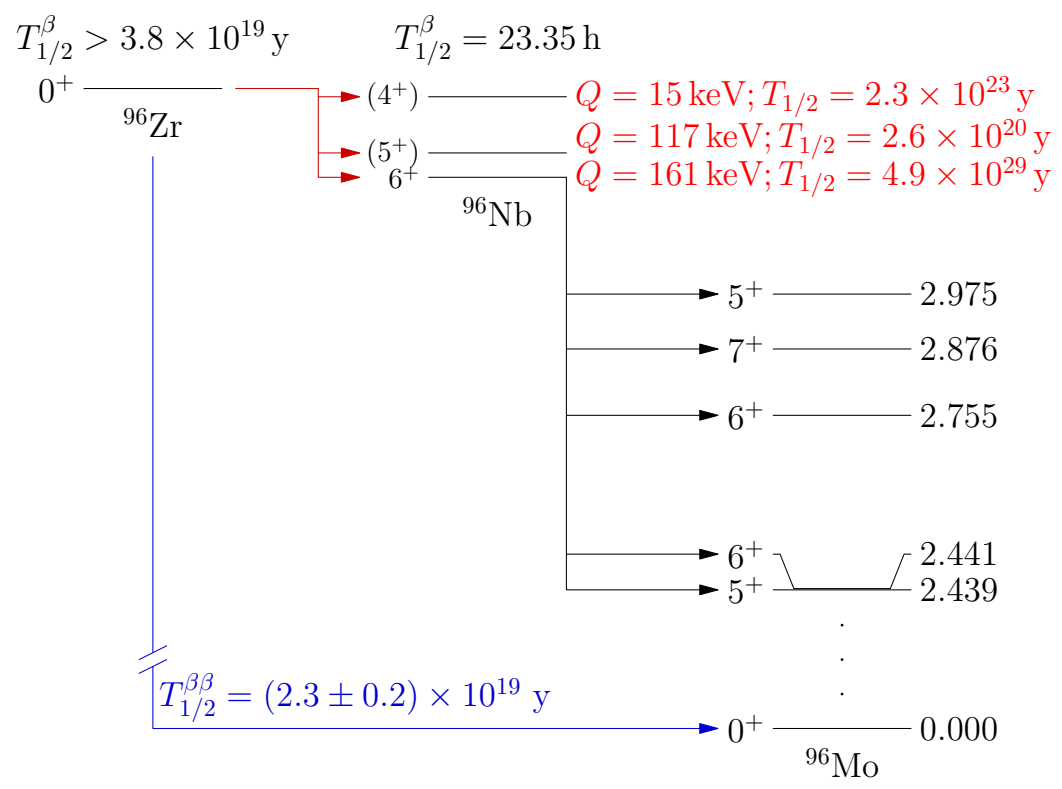

Figure 5. Ultra-slow beta-decay transitions from the ground state of ${ }^{96} \mathrm{Zr}$ to the three lowest states in ${ }^{96} \mathrm{Nb}$, and the subsequent beta decay to states in ${ }^{96} \mathrm{Mo}$. The experimental $Q$ values and computed half-lives are given to the right of the energy levels and the experimental lower limit of the total $\beta^{-}$decay half-life is given in the up left corner of the figure. Shown is also the experimental half-life of the direct double-beta-decay transition to the ground state of ${ }^{96} \mathrm{Mo}$. The numbers to the right of the ${ }^{96} \mathrm{Mo}$ energy levels are excitation energies in $\mathrm{MeV}$.

Let us now discuss an example pertaining to the points (b) and (c) of the introduction. In figure 5 the mother nucleus ${ }^{96} \mathrm{Zr}$ decays to states in ${ }^{96} \mathrm{Nb}$ via ultra-slow beta transitions, retarded by the large differences in angular momentum between the initial state (spin 0) and the final states (spins 4-6), belonging to the category (b) of the classification of ultra-slow decay transitions. In addition to the 
ultra-slow beta transitions there is a very interesting ultra-slow direct transition from ${ }^{96} \mathrm{Zr}$ to the ground state of ${ }^{96} \mathrm{Mo}$. In this case the decay jumps past the nucleus ${ }^{96} \mathrm{Nb}$ and goes directly to the ground state of ${ }^{96} \mathrm{Mo}$ and thus it falls into the category (c) in our classification of ultra-slow processes. These higher-order transitions form a class of transitions called generically the nuclear double beta decay [1].

The half-lives of figure 5 have been calculated [21] by using the experimental $Q$ values listed in the figure by the use of the proton-neutron QRPA (quasiparticle random-phase approximation). A similar situation as here occurs also for the beta and double beta decays of ${ }^{48} \mathrm{Ca}$. These decays have been discussed in [22] by the use of the nuclear shell model. In the present case the total beta-decay halflife $T_{1 / 2}\left(\beta^{-}\right)=2.6 \times 10^{20} \mathrm{y}$ is determined by the fourth-forbidden unique $\beta^{-}$transition to the $5^{+}$state in ${ }^{96} \mathrm{Nb}$. The other transitions, the fourth-forbidden non-unique transition to the $4^{+}$state and the sixthforbidden non-unique transition to the $6^{+}$state, do not play a role in the total half-life due to their very long partial half-lives. In fact, it is interesting to note that the computed $\beta^{-}$-decay half-life is an order of magnitude longer than the experimental double beta half-life $T_{1 / 2}\left(\beta^{-} \beta^{-}\right)=(2.3 \pm 0.2) \times 10^{19} \mathrm{y}[23]$. This bears relevance to the speculated time variation of the weak-interaction constant [24] noticed by comparing the modern laboratory-measured half-lives of double-beta nuclei with the geochemically obtained half-lives of the same nuclei, obtained by applying chemisty on very old terrestial ores [24]. The present result suggests that no severe interference of the $\beta^{-}$decay is expected in the $\beta^{-} \beta^{-}$-decay half-life of the old ${ }^{96} \mathrm{Zr}$ ores.

\section{Resonant neutrinoless double electron capture}

Neutrinoless double beta decay involves Majorana neutrinos as propagator between the two decay vertices and thus there are no (anti)neutrinos in the final state [1]. On the double $\beta^{-}$side the final state involves two emitted electrons and on the double $\beta^{+}$side there are two positrons in the final state [1]. Also the neutrinoless $\beta^{+} \mathrm{EC}$ decay occurs via the one-positron phase space. In the case of the neutrinoless double electron capture, $0 v \mathrm{ECEC}$, there are no leptons available in the final state to carry away the decay energy. In this case one has to engage some additional mechanism to rid the initial atom of the excess energy of decay. There are two proposed mechanisms to cope with this situation: the radiative $0 v \mathrm{ECEC}$ decay [25] and the resonant decay, R0vECEC [26]. The resonance condition close degeneracy of the initial and final (excited) atomic states - can enhance the decay rate by a factor as large as $10^{6}$. The R $0 v$ ECEC process is of the form

$$
\mathrm{e}^{-}+\mathrm{e}^{-}+(\mathrm{A}, \mathrm{Z}) \rightarrow(\mathrm{A}, \mathrm{Z}-2)^{*} \rightarrow(\mathrm{A}, \mathrm{Z}-2)+\gamma+2 \mathrm{X}
$$

where the capture of two atomic electrons leaves the final atom in an excited state, in most cases having the final nucleus in an excited state. The excited state of the nucleus decays by one or more gamma-rays and the atomic vacancies are filled by outer electrons with emission of X-rays.

Fulfillment of the resonance condition depends on the so-called degeneracy parameter $Q-E$, where $E$ is the excitation energy of the final atomic state and $Q$ is the difference between the initial and final atomic masses. Also the nuclear structure is heavily involved through the appropriate nuclear matrix elements [27]. Possible candidates for such resonant decays are many and a representative list is displayed in Table 2

In the table we also list the estimated half-lives for the cases for which such exist. The references of the last column indicates the origin of the $Q$-value measurement and the possible calculations of the related NME. The $Q$-value measurements have been performed by the use of modern Penning-trap techniques. In the table the quantity $C^{\mathrm{ECEC}}$ is listed and it relates to the R0vECEC half-life as given 
Table 2. R0 $v$ ECEC decay transitions with the final-state spin-parity indicated in the second column and the degeneracy parameters $Q-E$ in the third column. The involved atomic orbitals have been given in the fourth column. The second last column lists the currently available half-live estimates with the references to the $Q$-value measurement and calculations indicated in the last column.

\begin{tabular}{cccccl}
\hline Transition & $J_{f}^{\pi}$ & $Q-E[\mathrm{keV}]$ & Orbitals & $C^{\text {ECEC }}$ & Ref. \\
\hline${ }^{74} \mathrm{Se} \rightarrow{ }^{74} \mathrm{Ge}$ & $2^{+}$ & 2.23 & $\mathrm{~L}_{2} \mathrm{~L}_{3}$ & $(0.2-100) \times 10^{43}$ & {$[28]$} \\
${ }^{96} \mathrm{Ru} \rightarrow{ }^{96} \mathrm{Mo}$ & $2^{+}$ & $8.92(13)$ & $\mathrm{L}_{1} \mathrm{~L}_{3}$ & & {$[29]$} \\
& $0^{+} ?$ & $-3.90(13)$ & $\mathrm{L}_{1} \mathrm{~L}_{1}$ & & \\
${ }^{102} \mathrm{Pd} \rightarrow{ }^{102} \mathrm{Ru}$ & $2^{+}$ & $75.26(36)$ & $\mathrm{KL}_{3}$ & & {$[30]$} \\
${ }^{106} \mathrm{Cd} \rightarrow{ }^{106} \mathrm{Pd}$ & $0^{+} ?$ & 8.39 & $\mathrm{KK}$ & $(2.1-5.7) \times 10^{30}$ & {$[31]$} \\
& $(2,3)^{-}$ & $-0.33(41)$ & $\mathrm{KL}_{3}$ & & {$[30]$} \\
${ }^{112} \mathrm{Sn} \rightarrow{ }^{112} \mathrm{Cd}$ & $0^{+}$ & -4.5 & $\mathrm{KK}$ & $>5.9 \times 10^{29}$ & {$[32]$} \\
${ }^{124} \mathrm{Xe} \rightarrow{ }^{124} \mathrm{Te}$ & $0^{+} ?$ & $1.86(15)$ & $\mathrm{KK}$ & $(1.7-5.1) \times 10^{29}$ & {$[33]$} \\
${ }^{130} \mathrm{Ba} \rightarrow{ }^{130} \mathrm{Xe}$ & $0^{+} ?$ & $10.18(30)$ & $\mathrm{KK}$ & & {$[33]$} \\
${ }^{136} \mathrm{Ce} \rightarrow{ }^{136} \mathrm{Ba}$ & $0^{+}$ & -11.67 & $\mathrm{KK}$ & $(3-23) \times 10^{32}$ & {$[34]$} \\
${ }^{144} \mathrm{Sm} \rightarrow{ }^{144} \mathrm{Nd}$ & $2^{+}$ & $171.89(87)$ & $\mathrm{KL}_{3}$ & & {$[30]$} \\
${ }^{152} \mathrm{Gd} \rightarrow{ }^{152} \mathrm{Sm}$ & $0_{\mathrm{gs}}^{+}$ & $0.91(18)$ & $\mathrm{KL}_{1}$ & $(1.0-1.5) \times 10^{27}$ & {$[35,36]$} \\
${ }^{156} \mathrm{Dy} \rightarrow{ }^{156} \mathrm{Gd}$ & $1^{-}$ & $0.75(10)$ & $\mathrm{KL}_{1}$ & & {$[37]$} \\
& $0^{+}$ & $0.54(24)$ & $\mathrm{L}_{1} \mathrm{~L}_{1}$ & & {$[37]$} \\
& $2^{+}$ & $0.04(10)$ & $\mathrm{M}_{1} \mathrm{~N}_{3}$ & & {$[37]$} \\
${ }^{162} \mathrm{Er} \rightarrow{ }^{162} \mathrm{Dy}$ & $2^{+}$ & $2.69(30)$ & $\mathrm{KL}_{3}$ & & {$[29]$} \\
${ }^{164} \mathrm{Er} \rightarrow{ }^{164} \mathrm{Dy}$ & $0_{\mathrm{gs}}^{+}$ & $6.81(13)$ & $\mathrm{L}_{1} \mathrm{~L}_{1}$ & $(3.2-5.2) \times 10^{31}$ & {$[36,38]$} \\
${ }^{168} \mathrm{Yb} \rightarrow{ }^{168} \mathrm{Er}$ & $\left(2^{-}\right)$ & $1.52(25)$ & $\mathrm{M}_{1} \mathrm{M}_{3}$ & & {$[29]$} \\
${ }^{180} \mathrm{~W} \rightarrow{ }^{180} \mathrm{Hf}$ & $0_{\mathrm{gs}}^{+}$ & $11.24(27)$ & $\mathrm{KK}$ & $(4.0-9.5) \times 10^{29}$ & {$[36,39]$} \\
\hline
\end{tabular}

by

$$
T_{1 / 2}^{\mathrm{R} 0 v \mathrm{ECEC}}=\frac{C^{\mathrm{ECEC}}}{\left(m_{\mathrm{eff}}[\mathrm{eV}]\right)^{2}} \text { years }
$$

where the effective neutrino mass should be given in units of eV. In all the listed cases where $C^{\mathrm{ECEC}}$ has been computed the decay rates are suppressed by the rather sizable magnitude of the degeneracy parameter. Decays to $0^{+}$states are favored over the decays to $2^{+}$or $1^{-}, 2^{-}, 3^{-}$etc. states due to the involved nuclear wave functions and/or higher-order transitions. Also captures from atomic orbitals with orbital angular momentum $l>0$ are suppressed [28].

There are some favorable values of degeneracy parameters listed in Table 2, like ${ }^{106} \mathrm{Cd} \rightarrow$ ${ }^{106} \mathrm{Pd}(2,3)^{-}$and ${ }^{156} \mathrm{Dy} \rightarrow{ }^{156} \mathrm{Gd}\left(0^{+}, 1^{-}, 2^{+}\right)$but the associated nuclear matrix elements are not yet evaluated. At the moment the most favorable case with a half-life estimate is the case ${ }^{152} \mathrm{Gd} \rightarrow$ ${ }^{152} \mathrm{Sm}\left(0_{\mathrm{gs}}^{+}\right)$which describes a decay transition to the ground state.

\section{References}

[1] J. Suhonen, O. Civitarese, Phys. Rep. 300, 123 (1998)

[2] J. Suhonen, O. Civitarese, J. Phys. G: Nucl. Part. Phys. 39, 085105 (2012)

[3] J. Suhonen, O. Civitarese, J. Phys. G: Nucl. Part. Phys. 39, 124005 (2012)

[4] F.T. Avignone III, S.R. Elliott, J. Engel, Rev. Mod. Phys. 80, 481 (2008) 
[5] F. Schwamm, AIP Conf. Proc. 605, 461 (2002)

[6] G. Drexlin, V. Hannen, S. Mertens, C. Weinheimer, Advances in High Energy Physics 2013, 293986 (2013)

[7] H. Behrens, W. Bühring, Electron Radial Wave Functions and Nuclear Beta decay (Clarendon, Oxford, 1982)

[8] M.T. Mustonen, M. Aunola, J. Suhonen, Phys. Rev. C 73, 054301 (2006)

[9] M.T. Mustonen, J. Suhonen, Phys. Lett. B 657, 38 (2007)

[10] E. Ydrefors, M.T. Mustonen, J. Suhonen, Nucl. Phys. A 842, 33 (2010)

[11] B.J. Mount, M. Redshav, E.G. Myers, Phys. Rev. Lett. 103, 122502 (2009)

[12] J.S.E. Wieslander, J. Suhonen, T. Eronen, M. Hult, V. Elomaa, A. Jokinen, G. Marissens, M. Misiaszek, M.T. Mustonen, S. Rahaman et al., Phys. Rev. Lett. 103, 122501 (2009)

[13] C.M. Cattadori, M. De Deo, M. Laubenstein, L. Pandola, V.I. Tretyak, Nucl. Phys. A 748, 333 (2005)

[14] M.T. Mustonen, J. Suhonen, J. Phys. G 37, 064008 (2010)

[15] J. Suhonen, M.T. Mustonen, Prog. Part. Nucl. Phys. 64, 235 (2010)

[16] M.T. Mustonen, J. Suhonen, Phys. Lett. B 703, 370 (2011)

[17] N. Sugarman, Phys. Rev. 75, 1473 (1949)

[18] B. Singh, A.A. Rodionov, Y.L. Khazov, Nucl. Data Sheets 109, 517 (2008)

[19] M. Haaranen, J. Suhonen, Eur. Phys. J. A 49: 93 (2013)

[20] M.T. Mustonen, Private communication

[21] H. Heiskanen, M.T. Mustonen, J. Suhonen, J. Phys. G 34, 837 (2007)

[22] M. Aunola, J. Suhonen, T. Siiskonen, Europhys. Lett. 46, 577 (1999)

[23] A.S. Barabash, Phys. Rev. C 81, 035501 (2010)

[24] A.S. Barabash, Eur. Phys. J. A 8, 137 (2000)

[25] Z. Sujkowski, S. Wycech, Phys. Rev. C 70, 052501 (2004)

[26] J. Bernabeu, A. De Rujula, C. Jarlskog, Nucl. Phys. B 223, 15 (1983)

[27] J. Suhonen, Eur. Phys. J. A 48: 51 (2012)

[28] V. Kolhinen, V. Elomaa, T. Eronen, J. Hakala, A. Jokinen, M. Kortelainen, J. Suhonen, J. Äystö, Phys. Lett. B 684, 17 (2010)

[29] S. Eliseev et al., Phys. Rev. C 83, 038501 (2011)

[30] M. Goncharov et al., Phys. Rev. C 84, 028501 (2011)

[31] J. Suhonen, Phys. Lett. B 701, 490 (2011)

[32] S. Rahaman, V. Elomaa, T. Eronen, J. Hakala, A. Jokinen, A. Kankainen, J. Rissanen, J. Suhonen, C. Weber, J. Äystö, Phys. Rev. Lett. 103, 042501 (2009)

[33] D.A. Nesterenko et al., Phys. Rev. C 86, 044313 (2012)

[34] V.S. Kolhinen, T. Eronen, D. Gorelov, J. Hakala, A. Jokinen, A. Kankainen, J. Rissanen, J. Suhonen, J. Äystö, Phys. Lett. B 697, 116 (2011)

[35] S. Eliseev et al., Phys. Rev. Lett. 106, 052504 (2011)

[36] T.R. Rodríguez, G. Martínez-Pinedo, Phys. Rev. C 85, 044310 (2012)

[37] S. Eliseev et al., Phys. Rev. C 84, 012501(R) (2011)

[38] S. Eliseev et al., Phys. Rev. Lett. 107, 052501 (2011)

[39] A. Droese et al., Nucl. Phys. A 775, 1 (2012) 